\title{
SURGERY OF THE THYMUS
}

\author{
J. KeITH Ross, M.S., F.R.C.S. \\ Harefield Hospital, Middlesex and Middlesex Hospital, W.1
}

THE EXACT funotion of the thymus gland is still not understood. Within the last three or four years it has been discovered that the thymus is connected with the mammalian immune mechanism (Miller, 1963; Burnet, 1962).

Evidence that the thymus may be involved as part of the disease process in several conditions of obscure aetiology, including myasthenia gravis, some or all of which have an underlying disturbance of the immune response, offers real hope that a clearer understanding of the value of thymectomy in such conditions may be reached before very long. If this is so it may be possible to consolidate the position already held by thymectomy, give precision to its application and rid the operation of its empirical reputation.

\section{Thymic Tumours}

Tumours of the thymus and their management may be properly considered as a separate problem. When found in association with specific clinical syndromes, it is the tumour that dictates the tactics of treatment.

\section{Pathology}

The descriptive histological classification of Thomson and Thackray (1957) has the great merit that it can be related to the clinical course of the disease thus helping both orderliness of diagnosis and the prognosis. The classification is as follows:-

\section{Epithelial}

(a) Differentiated or epidermoid

(b) Oval or spindle cell

(c) Lympho-epithelioma

(d) Granulomatous

(e) Undifferentiated

II. Lymphoid (Lymphosarooma)

III. Teratomatous

Of the epithelial group, the oval cell and lymphoepitheliomatous tumours are encapsulated and apparently benign. The differentiated epithelial tumours, at first encapsulated, later prove locally invasive and finally may metastasize, but rarely to sites outside the chest. The granulomatous thymoma is not often found in its early and circumscribed state and histologically resembles Hodgkin's disease: a tendency to early infiltration of neighbouring vital structures and to produce early lymph node metastases is to some extent offset by its considerable radiosensitivity. Lymphosarcoma, undifferentiated carcinoma and teratoma - the latter a disease of the young adult male-are uniformly highly leihal. The incidence of myasthenia gravis is greatest amongst tumours at the benign end of the behaviour-range, almost exclusively lymphoepitheliomata.

\section{Diagnosis}

Clinical presentation-Unless the tumour occurs in association with myasthenia gravis or other associated clinical syndromes, it is either dis- $\overrightarrow{\vec{\omega}}$ covered accidentally (routine chest radiograph) or ${ }_{\circ}$ gives rise to symptoms and signs indicating that it $\bar{D}$ is inoperable. Genuine retrosternal pain is an ominous symptom. Superior vena caval obstruction, pericardial or pleural effusions, phrenic nerve $\stackrel{\vec{N}}{\mathrm{f}}$ palsy, and malignant lymph nodes in the neck all $\vec{\infty}$ indicate degrees of spread that rule out surgery as the primary mode of treatment.

Radiological diagnosis may be supplemented by

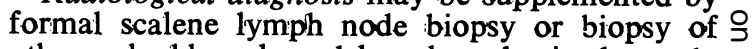
other palpably enlarged lymph nodes in the neck. Mediastinal biopsy, by resection of the appropriate $D$ second costal cartilage and an extrapleural approach to the mass may also give useful informa- $\vec{\bullet}$ tion. Mediastinoscopy (Nohl-Oser, 1965) is n\& $\mathscr{\circ}$ useful unless the tumour is malignant and ha metastasized to the paratracheal lymph nodes. because this investigation is carried out in a plane deep to the aortic arch and the left innominate vein. When pleural or pericardial effusions have developed, cytology of the fluid may give positive results.

\section{Treatment}

The malignant thymoma that presents with signs of advanced spread should be treated by irradiation without delay. A strong case can be made for residual thymectomy after radiotherapy; viable tumour cells embedded in the fibrous remains of the gland have been found sufficiently often to make this a reasonable proposition in appropriate cases (Sellors, 1961).

In the absence of tumour spread outside the anterior mediastinum, the initiative lies with the 9 surgeon. In such instances (without myasthenia) there is often doubt about the exact diagnosis and a policy of routine preoperative irradiation will result in a proportion receiving unnecessary treat- O ment: this is undesirable.

When the problem is approached surgically, three possible situations may be met.

First, a localized, well-encapsulated tumour may be found in a thymus that is easy to remove together with the other contents of the anterior mediastinum. A radical clearance of the anterior mediastinum is all that is needed in such instances.

Secondly, the tumour may prove to be one of 
spread has taken place it is not so advanced that radical excision is prevented. The favourable the malignant variants at an early stage or if local results of such radical surgery indirate that it is justifiable to include, if need be, pericardium, pleura, invaded lung, phrenic nerve and left innominate vein in the clearance (Le Roux, 1961). In such instances, postoperative irradiation should be given whether or not excision appears complete from examination of the specimen.

Thirdly, it may be found that intrathoracic spread has taken place to a degree not suspected on clinical or radiological grounds. If this is so, the tumour may be judged inoperable and biopsied, but there is much to be said for removing as much as possible of the bulk of the tumour without endangering the patient's life. In contrast to many maligant tumours, partial removal followed by radiotherapy can give surprisingly good results. Whether this is entirely due to the radiosensitivity of thymic tumours, whether unexpected benefit follows the sudden reduction of tumour mass within the host, or whether the result reflects some innate property of the tumour are all matters for speculation.

\section{Thymectomy and Myasthenia Gravis}

It has been known since the turn of the century that a connection exists between myasthenia gravis and abnormalities of the thymus gland (Blalock, Mason, Morgan, and Liden, 1939), and the main interest lies in the results of thymectomy in this disease.

In the last decade, large series of thymectomies for myasthenia have been reported, notably by Sir Geoffrey Keynes $(1946,1955)$ and by Clagett at the Mayo Clinic (Eaton and Clagett, 1955). The late results of the former have been examined by Simpson (1958), and those of a smaller series recently published largely agree with their forerunners (Henson, Stern, and Thompson, 1965).

Unless they have a thymoma, patients under the age of 45-50 with generalized myasthenia are likely to do better with a thymectomy than without, regardless of their sex or the duration of their symptoms. Women who are young when the disease first appears and who are operated upon early obtain most benefit, and older males least. The results are best, and the saving of life is greatest when the duration of the disease is less than five years and no thymoma is present. Myasthenia of over seven years' duration is less likely to respond favourably to thymectomy, but a long history does not preclude some improvement, although cure is unlikely.

By comparing the mortality in groups of patients treated surgically with others treated medically, it has been shown that thymectomy has been responsible for a significant saving of life. Further, 50 to 60 per cent of operated cases (non-tumour) are very greatly improved compared with the spontaneous remission rate of 20 to 25 per cent in those treated medically. The severity of the myasthenia preoperatively does not influence the result in most instances. Although there is general agreement that females ( 53 per cent) benefit more than males ( 35 per cent) the figures suggest that the general trend for both sexes is a beneficial one. The operation should not, therefore, be withheld from men merely because their improvement is less dramatic.

Myasthenia gravis is well known for its characteristic remissions, and many of the milder cases who are able to carry on an active and useful life with the help of drugs in small dosages are never seen by the surgeon. Others have their myasthenia confined to localized groups of muscles (e.g. the external ocular muscles) and surgery is seldom if ever indicated in either of these situations (Ferguson, 1962). Improvement rarely follows thymectomy when the muscle weakness is static and has been present for a long time. Extensive muscle wasting (not usually a feature of myasthenia) or serious associated and coincidental disorders can also be contraindications to operation.

The presence of a thymic tumour, found in 10 to 20 per cent of myasthenies, is no contraindication to operation-rather the reverse. Although in Simpson's review 60 to 70 per cent of those with tumours proved to have died or deteriorated, and in Clagett's series 58 per cent with thymomas died (chiefly of myasthenia) compared with an overall death rate of 30 per cent for his whole series, the improvement in the myasthenia in the 18 to 20 per cent who do improve can be as great as that seen in the nontumour cases. Moreover, removal of the tumour is justifiable in its own right.

\section{Autoimmunity and Myasthenia Gravis}

In a study of 74 myasthenics investigated for evidence of associated autoimmune disease, autoantibodies were found in 77 per cent (Downes, Greenwood, and Wray, 1965). Thymectomy was found to have little influence on the incidence of autoantibodies, nor was there any significant correlation between their incidence and the response to thymectomy. Sufficient evidence was collected, however, for the authors to claim an increased frequency of autoimmune disease in patients with myasthenia gravis. A more farreaching suggestion is also made by the same workers. They postulate that an increased rate of somatic mutation within the thymus, favoured by its rapid rate of cell-division, might lead both to abnormal immunological reactions and to neoplastic transformation. This might account for the frequent association of thymic tumours and myasthenia gravis and for the occurrence of such tumours in patients with other conditions thought to have an autoimmune basis. These include systemic lupus erythematosus, Sjogren's syndrome, rheumatoid arthritis, Addison's disease and autoimmune haemolytic anaemia. Thymectomy has been tried in systemic lupus erythematosus (Mackay, Goldstein, and McConchie, 1963). 


\section{Clinical Syndromes Associated with Thymic Tumours}

The following clinical syndromes have been described in association with thymic tumours, sometimes combined with myasthenia.

(i) Red Cell Aplasia

Pure red cell aplasia may exist alone or be combined with abnormal white cell and platelet counts (Norman, 1965). The results of thymectomy are inconsistent, and it seems certain that both the tumour and the anaemia are produced by something which has not yet been discovered. The tumours themselves are usually benign.

(ii) Hypogammaglobulinaemia

This complication is found in about 6 per cent of thymomas which are usually benign lymphoepitheliomas or spindle cell tumours (Godfrey, 1964). The results of thymectomy, again, are variable (Maldonado, Bayrd, and Kiely, 1964).

(iii) Cushing's Syndrome

Although Cushing's syndrome is commoner in women, when found in association with a thymic tumour it appears to be commoner in males (Scholz, and Bahn, 1959) and the tumour is more frequently malignant than in any of the other associated clinical entities. There is an inverse relationship between abnormal adrenal cortical activity and the size of the thymus, and in Cushing's syndrome with associated thymoma it is usual to find little or no thymic tissue surrounding the tumour. A variable response is obtained if ACTH is given in myasthenia (Adams, DennyBrown, and Pearson, 1953).

Finally, the relationship between myasthenia gravis and thyroid disease (Sahay, Blendis, and Greene, 1965) and the fascinating problem of neonatal myasthenia with its possible bearing on the aetiology of the disease (Stern, Hall, and Robinson, 1964) deserve mention (as two further facets of a disease complex that is emerging before our eyes.

\section{REFERENCES}

Adams, R. D., Denny-Brown, D., and Pearson, C. M. (1953): Diseases of Muscle. p. 491. London: Cassell.

Blalock, A., Mason, M. F., Morgan, H. J. and RIVEN, S. S. (1939): Myasthenia Gravis and
Tumours of the Thymic Region, Ann. Surg., 110, 544.

BURNET, Sir Macfarlane. (1962): Role of the Thymus and Related Organs in Immunity, Brit. med. J., ii, 5308 .

Downes, J. M., Greenwood, B. M., and Wray, S. H. (1965): Autoimmune Aspects of Myasthenia Gravis. Quart. J. Med., 35, 85.

Eaton. L. M. and Clagett. O. T. (1955): The Present Status of Thymectomy in the Treatment of Myasthenia Gravis, Amer. J. Med., 19, 703.

Ferguson, F. R. (1962): A Critical Review of the Clinical Features of Myasthenia Gravis, Proc. roy. Soc. Med., 55, 49.

GoDfreY, S. (1964): Thymoma with Hypogammaglobulinaemia in an Identical Twin, Brit. med. J., $i$, 1159.

Henson, R. A., Stern, G. M., and Thompson, V. C. (1965): Thymectomy for Myasthenia Gravis, Brain, 88,11 .

Keynes, Sir Geoffrey, L. (1946): The Surgery of the Thymus Gland, Brit. J. Surg., 33, 201.

- (1955): Investigations into Thymic Disease and Tumour Formation, Brit. J. Surg., 42, 449.

LE Roux, B. T. (1961): A Clinical Appraisal of Thymic Lesions and Anterior Mediastinal Lymphomata, Thorax, 16, 156.

iMcKaY, I. R., GoldSTEIN, G., and McConchie, I. H. (1963): Thymectomy in Systemic Lupus Erythematous, Brit. med. J., ii, 792.

Maldonado, J. E., BaYRd, E. D., and Kiely, J. M. (1964): The Thymus Gland and its Relationship to the Haemopoietic and Immunologic Systems: A Review, Proc. Mayo Clinic, 39, 60.

Miller, J. F. A. P. (1963): Role of the Thymus in Immunity, Brit. med. J., ii, 459.

NOHL-OSER, H. C. (1965): Mediastinoscopy, Brit? med. J., i, 1167.

Norman, A. G. (1965): Thymic Tumours with Red Cell Aplasia, Thorax, 20, 193.

Sahay, B. M., Blendis, L. M., and Greene, R. (1965): Relation Between Myasthenia Gravis and Thyroid Disease, Brit. med. J., i, 762.

Scholtz, D. A., and BaHN, R. C. (1959): Thymic Tumours Associated with Cushing's Syndrome: Review of Three Cases, Proc. Mayo Clin., 34, 433.

SELloRS, T. H. (1961): Surgery of the Thymus Gland, Bull. int. Chir., 20, 315.

SiMPSON, J. A. (1958): An Evaluation of Thymectomy in Myasthenia Gravis, Brain, 81, 112.

Stern, G. M., Hall, J. M., and Robinson, D. C. (1964): Neonatal Myasthenia Gravis, Brit. med. J., ii, 284.

Thomson, A. D., and Thackray, A. C. (1957): The Histology of Tumours of the Thymus, Brit. J. Cancer, 11, 348. 